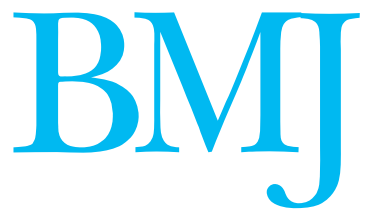

\title{
Pre-eclampsia matters
}

\author{
New guideline is simple, evidence based, and clinical, and should be used
}

Papers p 565 Primary care p 576
$\mathrm{P}$ re-eclampsia matters. In both the developed and the developing world, pre-eclampsia is important. It remains a leading cause of maternal and perinatal mortality and extensive morbidity. The reports of the Confidential Enquiry Into Maternal Deaths ${ }^{1}$ have identified deficiencies in care in relation to pre-eclampsia in successive reports since the 1950s. In this issue, the systematic review by Duckitt and Harrington quantifies the risk of pre-eclampsia associated with different factors present at the antenatal booking visit (p 565). ${ }^{2}$ The rationale is that this risk assessment will inform allocation of the woman to a suitable surveillance routine to detect pre-eclampsia.

The risk of pre-eclampsia is increased with a previous history of pre-eclampsia, pre-existing diabetes, multiple pregnancy, a family history for pre-eclampsia, a raised body mass index before pregnancy or at booking, raised blood pressure at booking, and the presence of anti-phospholipid antibodies. These risk factors are important, with relative risks of almost threefold for nulliparity and over ninefold for antiphospholipid antibodies. With a background incidence of 2-3\%, this translates to absolute risks of $6 \%$ and almost $30 \%$, respectively. In terms of early onset of disease, which carries the highest level of morbidity, the authors note that they may have underestimated the importance of these risk factors as studies often did not discriminate between early and late onset pre-eclampsia. Further, they could not study the interrelations between risk factors-for example, obesity-maternal age, and essential hypertension.

Clinicians spend a great deal of time and energy screening for problems in pregnancy-for example, syphilis, Rhesus iso-immunisation, and Down's syndrome-that have a much lower incidence than pre-eclampsia. So why have we failed when the identification of women at risk, and the diagnosis of pre-eclampsia, through measurement of blood pressure and urinalysis, is arguably the most important aspect of regular antenatal assessment? Complacency may play a part, as clinicians and women perhaps take for granted a healthy outcome of pregnancy, but we have undoubtedly failed to appreciate the risk factors, the protean presentations of this condition, its natural history, and the need for intervention. Many women do not realise why they are having their blood pressure measured. In addition, clinicians may see risk assessment and screening for pre-eclampsia as futile. The criteria for screening are that the condition must relate to an important health problem; the natural history should be understood and identifiable before the disease presents clinically; the screening test should be simple, safe, and acceptable; effective treatment must exist, with better outcomes than late treatment; and the treatment should be effective in reducing morbidity and mortality, acceptable, and cost effective. Not all these criteria are met for preeclampsia. However, the quantification of risk for different factors provided by this systematic review is welcomed. ${ }^{2}$

These evidence based risk factors have been used by the Pre-Eclampsia Community Guideline (PRECOG) Group to inform its community guideline, which is also published in this issue ( $p$ 576). ${ }^{3}$ These factors will allow early referral and a two tier schedule of assessment for signs of pre-eclampsia, complementing the NICE antenatal guideline. ${ }^{4}$ That absence of antenatal care is associated with poor outcome of pregnancy is now clear, ${ }^{5}$ but, perhaps surprisingly, we have little evidence to support the optimal frequency of antenatal care visits for identifying pre-eclampsia. In many patients it develops rapidly and can progress to a severe form within days of an adequate antenatal assessment. In view of the rapid development of pre-eclampsia in some women, we need to ensure that antenatal education makes women aware of the symptoms of pre-eclampsia and the importance of regular assessment. The guideline is aimed at those caring for pregnant women in the community, to raise awareness of the risk factors for and implications of pre-eclampsia, including the need for accurate diagnosis, assessment, and timely referral especially when proteinuria or severe hypertension are present. Once severe disease develops, many women require delivery within three days, although with intensive control of blood pressure a mean prolongation of pregnancy of 14 days is possible. ${ }^{6}$ The PRECOG guideline adopts a pragmatic approach to tackle this issue, presenting a clear synthesis of the evidence, albeit limited in terms of randomised trials, and a clear plan setting out whom and when to refer.

The strengths of the PRECOG guideline are its simplicity, its evidence base for risk assessment, its reliance only on clinical features for assessment, and its straightforward management plan. These features make it relevant not only in the United Kingdom but also in other countries. The weakness is that the 
available evidence to support intervention with more frequent assessment or by different healthcare professionals is limited in quantity and quality.

Purists may argue that screening for risk in early pregnancy and recommendations for the proposed frequency of antenatal screening visits does not meet all the criteria for screening. However, in $46 \%$ of maternal deaths and $65 \%$ of fetal deaths reported via the confidential inquiries into maternal deaths, ${ }^{1}$ different management would reasonably have been expected to alter the outcome. Many of these management problems arise in the community because of a failure to identify and act on established risk factors at booking and to recognise and respond to signs and symptoms consistent with pre-eclampsia. We may not have the answers in terms of the outcome from such intervention, but we cannot be complacent in the face of the recurrent deficiencies identified in the confidential inquiries. The pragmatic approach of PRECOG is essential because pre-eclampsia matters.

Ian A Greer Regius professor of obstetrics and gynaecology

University of Glasgow, Glasgow Royal Infirmary, Glasgow G31 2ER

(I.A.Greer@clinmed.gla.ac.uk)

Competing interests: None declared.

1 Confidential Enquiry into Maternal Deaths. Why mothers die 2000-2002. The sixth report of the confidential enquiries into maternal deaths in the United Kingdom. London: Royal College of Obstetricians and Gynaecologists Press; 2004.

2 Duckitt K, Harrington D. Risk factors for pre-eclampsia at antenatal booking: systematic review of controlled studies. BMI 2005:330:565-7.

The pre-eclampsia community guideline (PRECOG): how to screen for The pre-eclampsia community guideline (PRECOG): how to screen for 80 .

4 National Institute for Clinical Excellence. NICE guideline CG6. Antenatal care-routine care for the healthy pregnant woman. London: NICE, 2003.

5 Abi Said D, Annegers JF, Combs Cantrell D, Frankowski RF, Willmore LJ. Case control study of the risk factors for eclampsia. Am J Epidemiol 1995; $142: 437-41$.

6 Sibai BM, Mercer BM, Schiff E, Friedman SA. Aggressive versus expectant management of severe pre-eclampsia 28 to 32 weeks gestation: a randomized controlled trial. Am J Obstet Gynecol 1994;171:818-22.

\title{
Counting the dead in Iraq
}

\author{
We need to know how many people have died
}

$\mathrm{C}$ ounting the dead is intrinsic to civilised society. Understanding the causes of death is a core public health responsibility. The government's white paper on public health emphasises the vital role of assessing the impact on health of all public policy. ${ }^{1}$ This is well recognised, and yet neither the public nor public health professionals are able to obtain reliable and officially endorsed information about the extent of civilian deaths attributable to the allied invasion of Iraq. Estimates vary between tens and hundreds of thousands.

These estimates come from reports in the press, or counting bodies admitted to hospitals, (www. iraqbodycount.net) as well as surveys. The former are likely to be inaccurate and to underestimate the true numbers and do not easily allow for reliable attribution between, for example, violent and natural causes. Public access to reliable data on mortality is important. The policy being assessed - the allied invasion of Iraq-was justified largely on grounds of democratic supremacy. Voters in the countries that initiated the war, and others-not least in Iraq itself-are denied a reliable evaluation of a key indicator of the success of that policy. This is unacceptable.

Instead the UK government's policy was first not to count at all, and then to rely publicly on extremely limited data available from the Iraqi Ministry of Health. This follows US government policy; famously encapsulated by General Tommy Franks of the US Central Command "We don't do body counts." Its inadequacy was emphasised after the publication of a representative household survey that estimated 100000 excess deaths since the 2003 invasion. ${ }^{3}$ The government rejected this survey and its estimates as unreliable; in part absurdly because statistical extrapolation from samples was thought invalid. ${ }^{4}$ Imprecise they are, but to a known extent. These are unique estimates from a dispassionate survey conducted in the most dangerous of epidemiological conditions. Hence the estimates, as far as they can go, are unlikely to be biased, even allowing for the reinstatement of Falluja. To confuse imprecision with bias is unjustified.

The methods for counting the dead in such circumstances are well established and cannot rely on incidental reports or assessments in hospital mortuaries alone. They require first hand verbal autopsies, ${ }^{5}$ which should be reliably obtained so that extrapolation to the population is possible, as Roberts et al had done. They also require some linkage with unclassified data on military offensives. ${ }^{6}$ Although active surveillance of this kind is extremely difficult in the context of such violence, even limited household surveys are essential so long as they are systematic. Such data can then be combined with information from passive sources to establish a more accurate overall assessment.

Counting casualties accurately can help to save lives both currently and in the future. Understanding the burden of death, injury, disease, and trauma that the population is currently suffering enables proper planning of war, and health, and in assessing local responses appropriately. In the future this should help government and military planners to assess the likely humanitarian implications of conflict.

The plain fact is that an estimate of 100000 excess deaths attributable to the invasion of Iraq is alarming. This is already half the death toll of Hiroshima. ${ }^{7}$ Apart from the practical arguments, the principled ones stand and will always stand. Have we not learnt any lessons from the history of sweeping alarming numbers of deaths under the carpet? This is not something about which there can be any political discretion 60 years after Auschwitz. The UK government, acting on our behalf, ought to offer reasoned criticism of the existing estimates. It should pursue their public health responsibilities to count the casualties by using modern methods. Democracy requires this, as does proper responsibility under the Geneva Conventions. See also News p 557 